\title{
Sampling Strategy for Ultrasonic Mid-Air Haptics*
}

\author{
William Frier ${ }^{\dagger}$ \\ University of Sussex \\ Brighton, United Kingdom \\ w.frier@sussex.ac.uk
}

\author{
Dario Pittera \\ University of Sussex \\ Brighton, United Kingdom
}

\author{
Damien Ablart \\ University of Sussex \\ Brighton, United Kingdom
}

\author{
Marianna Obrist \\ University of Sussex \\ Brighton, United Kingdom
}

\author{
Sriram Subramanian \\ University of Sussex \\ Brighton, United Kingdom
}
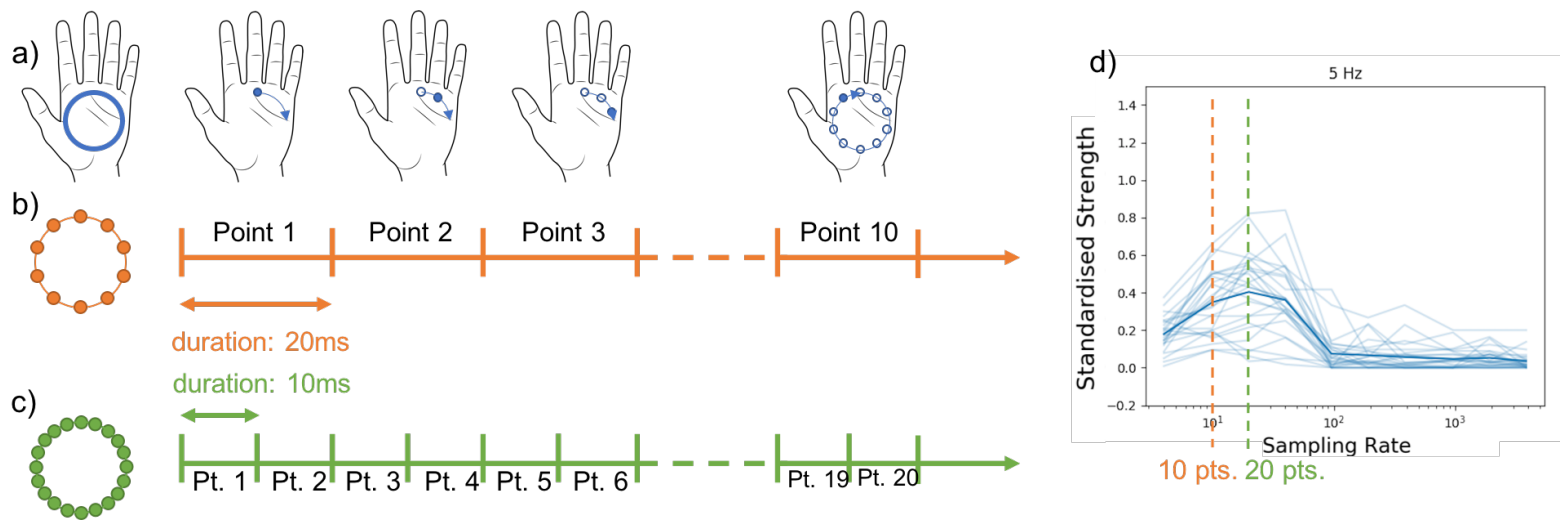

Figure 1: (a) A mid-air tactile pattern such as a circle is sampled into a set of successive positions, here 10. (b) Each sample point is presented during a given interval of time proportional to the total number of sample points. (c) Increasing the number of sample points will increase the rendering fidelity, but will also decrease the stimulation duration of each sample point. (d) Our study shows that changing the number of sample points affects the perceived strength of the pattern.

\begin{abstract}
Mid-air tactile stimulation using ultrasonics has been used in a variety of human computer interfaces in the form of prototypes as well as products. When generating these tactile patterns with mid-air tactile ultrasonic displays, the common approach has been to sample the patterns using the hardware update rate capabilities to their full extent. In the current study we show that the hardware update rate can impact perception, but unexpectedly we find that higher update rates do not improve pattern perception. In a first user study, we highlight the effect of update rate on the perceived strength
\end{abstract}

*Produces the permission block, and copyright information

$\dagger$ Also with Ultrahaptics Ltd..

Permission to make digital or hard copies of part or all of this work for personal or classroom use is granted without fee provided that copies are not made or distributed for profit or commercial advantage and that copies bear this notice and the full citation on the first page. Copyrights for thirdparty components of this work must be honored. For all other uses, contact the owner/author(s).

CHI 2019, May 4-9, 2019, Glasgow, Scotland Uk

(c) 2019 Copyright held by the owner/author(s).

ACM ISBN 978-1-4503-5970-2/19/05.

https://doi.org/10.1145/3290605.3300351 of a pattern, especially for patterns rendered at slow rate of less than $10 \mathrm{~Hz}$. In a second user study, we identify the evolution of the optimal update rate according to variations in pattern size. Our main results show that update rate should be designated as additional parameter for tactile patterns. We also discuss how the relationships we defined in the current study can be implemented into designer tools so that designers remain oblivious to this additional complexity.

\section{CCS CONCEPTS}

- Human-centered computing $\rightarrow$ Haptic devices.

\section{KEYWORDS}

Mid-air, Haptics

\section{ACM Reference Format:}

William Frier, Dario Pittera, Damien Ablart, Marianna Obrist, and Sriram Subramanian. 2019. Sampling Strategy for Ultrasonic Mid-Air Haptics. In CHI Conference on Human Factors in Computing Systems Proceedings (CHI 2019), May 4-9, 2019, Glasgow, Scotland Uk. ACM, New York, NY, USA, 11 pages. https://doi.org/10.1145/3290605. 3300351 


\section{INTRODUCTION}

Mid-air haptics is a growing field which aims to move the interaction from touch surfaces to touchless interactive spaces in 3D. Hence technologies such as air-jet [28], air-vortexes [24], laser[12, 22], electric arc [25], and ultrasonic-phased array $[3,7]$ were developed to provide mid-air tactile feedback to the user, without requiring augmentation with extra wearables. Among those technologies, ultrasonic phased arrays are particularly proficient at rendering a range of complex tactile patterns on the user's palm or fingertips. Indeed, the relatively small size of the tactile point produced (approximately the same as the carrier wavelength[9]) and the capability to render several points at once [3] has lead researchers to use ultrasonic phased arrays to generate tactile pattern such as volumetric shapes [16], tactile experiences [19] and even emotions [20].

Ultrasonic phased arrays focus sound waves coming from an array of ultrasonic transducers into a single location in space. In this focal region, the acoustic pressure almost instantly builds up and eventually becomes great enough to indent slightly the human skin and therefore stimulate the sense of touch. This focal region is thus equivalent to a tactile point. To convey a range of vibrotactile haptic perception through this tactile point, one needs to modulate the tactile point either in wave amplitude [7] also referred to Amplitude Modulation (AM), or in its lateral position [27] also referred to Lateral Modulation (LM). Obrist et al. has shown that varying the modulation frequency varied the perception of tactile point strength among other aspects of its perception [19] Another approach that constitutes an even more advanced modulation technique is to move the tactile point rapidly and repeatedly around a given path across the user's palm, hence producing a tactile pattern [13] (referred to SpatioTemporal Modulation (STM) ).

AM, LM, and STM are different ways of rendering tactile patterns and are the haptic analogues of graphical rendering methods on digital display screens. Like with most displays, ultrasonic phased arrays are controlled through a digital signal and therefore, each variation in pressure amplitude or spatial position is sampled across time. The rate at which the output is updated depends on the mid-air tactile display update rate capabilities and can range from $1 \mathrm{kHz}$ [27] to $20 \mathrm{kHz}$ [13]. As far as spatially distributed patterns are concerned, the device update rate can further be divided between, the number of sample to produce a single pattern iteration, and the rate at which the pattern shape is repeated. We referred to the former as pattern sampling rate and to the later as draw frequency .

However, little attention has been paid to update rates in mid-air haptics and, to the best of the authors knowledge, all published studies have used the maximal update rate the device has to offer to sample their output. Recall that in visual and auditory modalities a higher update rate improves the output quality by avoiding artefacts such as motion blur and flickering in vision and aliasing in audio. Drawing analogy with those modalities one has good reason to expect that a higher update rate will improve the quality of the mid-air tactile feedback.

However, by definition higher update rate leads to a shorter tactile stimulation duration per sample. Currently, the update rates of the ultrasonic phased array are above $1 \mathrm{kHz}[13,27]$ which is much greater than the maximum modulation frequency perceptible by our skin mechanoreceptors which is close to $500 \mathrm{~Hz}$ [10]. Such high update rates lead to sample times that are shorter than a millisecond. Contrast that to the temporal resolution of our sense of touch which is estimated to be between $2 \mathrm{~ms}$ and $40 \mathrm{~ms}$ [17]. In the same way that our hearing cannot listen to ultrasound frequencies above $20 \mathrm{kHz}$ or see subliminal images, it becomes unclear to what extent a user can perceive such rapid tactile stimulation.

In the current study, we answer this question by finding out if the update rate has an effect on the user perception of mid-air tactile patterns. To that end, we ran two user studies where mid-air tactile patterns are created with STM but using different draw frequencies and different sampling rates.

In the first user study, we show that the perceived strength of mid-air tactile pattern is related to the sampling rate of a pattern, especially for low draw frequencies (i.e. between $2 \mathrm{~Hz}$ and $10 \mathrm{~Hz}$ ). The results particularly show that for a high sampling rate (i.e. above 200 points), which is the common approach, patterns with low draw frequency (i.e. under $20 \mathrm{~Hz}$ ) could not be perceived by the user. However, using our approach, which is to lower the sampling rate, user could perceived pattern with draw frequency as low as $2 \mathrm{~Hz}$. Fitting our results to a quadratic mixed model, we were able to determine the relation between perceived strength and sampling rate, and further determine the the optimal sample rate that generates the strongest subjective perception of tactile feedback

In the second user study, we repeated the same method but instead varied the pattern sizes, e.g. the diameter of a circular pattern displayed on a user's palm as seen in Figure1. Combining these results with our first user study, we were therefore able to determine the optimal sample rate for each combination pattern size and pattern draw frequency. Our results show that this optimal sample rate is proportional to the pattern size. In other word, for a given draw frequency, one should optimise the sample rate according to the distance between two consecutive samples.

Finally we discuss the impact of our results on the design of tactile patterns and propose how our guidelines could be integrated within tactile feedback designer tools. 


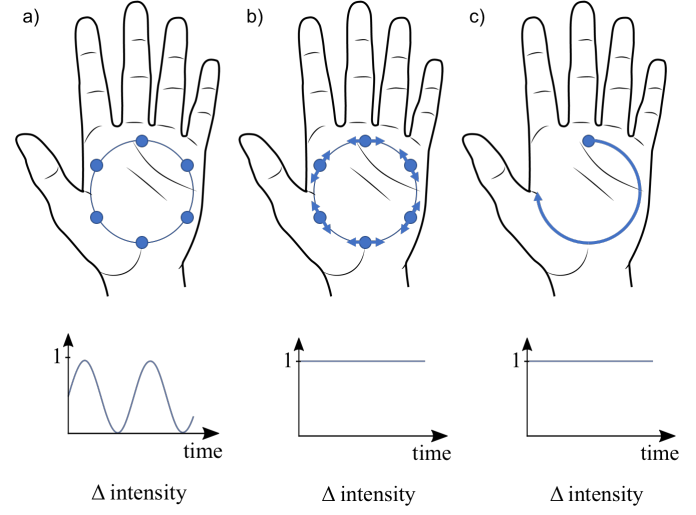

Figure 2: Mid-air tactile display can use 3 kinds of modulation techniques to produce a tactile pattern: (Left) Amplitude Modulation, (Middle) Lateral Modulation, (Right) Spatiotemporal Modulation. Each modulation technique varies the position and intensity of one or more mid-air tactile points differently over time.

\section{RELATED WORK}

Literature on mid-air tactile patterns is generally divided into two parts. Initially, most of the research focus was on advancing the hardware challenge and developed a functional system that can be electronically driven to produce mid-air tactile feedback. Once this important milestone was overcome, various application use cases were investigated including AR/VR, automotive interfaces and public displays. Recently, research focus has returned to investigate and further optimise the algorithms and methods that generate the tactile pattern in mid-air and the study effect of different rendering parameters on the overall perception. This paper is concerned with the latter.

\section{Mid-air Tactile Displays}

Researchers adopt different technologies and methods to develop mid-air tactile displays. One approach is to use pressurised air to convey tactile stimuli either in the form of air jets [28] or air-vortices [24]. This approach does not allow for multi-points stimulus. Furthermore air-jet stimulus size varies with the distance to the device, while air-vortices produce relatively big stimulus (few centimetres diameter).

Other approaches to produce mid-air tactile stimuli include the use of lasers [12,22] or the use of electric arcs [25]. Even though the stimulus size is very fine in such approaches, the interaction zone remains limited.

Another approach is to use an array of ultrasound transducer to convey tactile feedback in mid-air [7]. Indeed, by electronically shifting the transducer phases one could focus the acoustic pressure to a point in space and use it to produce tactile stimuli on the user hand. The force applied to the skin can reach $16 \mathrm{mN}$, for a contact area of $20 \mathrm{~mm}$ diameter [7].
More recently, with the use of $70 \mathrm{kHz}$ (instead of $40 \mathrm{kHz}$ ) ultrasound transducers one could produce even smaller focused points [9]. It was also shown how similar devices could be used to create multiple focus points with different tactile properties [3], or mixed with other mid-air tactile display [21], thus increasing the range of applications for HCI. Ultrasound phased arrays have therefore rapidly became a reliable and attractive technology for both researchers and developers interested in mid-air tactile applications.

As previously mentioned in the introduction, there are currently three different modulation techniques to generate a tactile perception in mid-air using an ultrasound phased array. These three modulation techniques are illustrated in Figure 2. Amplitude Modulation (AM) is the most commonly used modulation technique for mid-air tactile display and also the easiest to understand. AM involves modulating the pressure amplitude at the tactile point between 0 and 1 (its maximum output value) at a given periodic frequency. AM stimuli are therefore similar to vibrotactile stimuli that is commonly used in mechanical devices. Lateral Modulation (LM) is a more recent modulation technique where a tactile point oscillates back and forth along a short line that is parallel to the skin, while the focus acoustic pressure is fixed to 1 [27]. The authors of LM claim that this modulation technique generates a lateral force on the skin, which are usually perceived as being stronger than normal forces, and therefore is very different to AM. Spatiotemporal Modulation (STM) involves moving a tactile point rapidly and repeatedly along a path on the user's palm with the aim to induce the perception of a 2D shape [13]. This technique is similar to persistent of vision display, where spatial portions of the image are displayed successively and within the persistence time of the human eye, as to represent the whole graphic.

As AM and LM techniques only produce localized tactile effects and requires multi-points stimulus to produce spatially distributed patterns. On the other hand the STM technique is non-localized and requires only one point to produce spatially distributed patterns. Using a single tactile point generally allows for a stronger pressure output compared to many-points since the total hardware output power does not have to be shared by the different points as shown in Kappus and Long [13]. This paper focuses on STM methods to generate spatially distributed tactile patterns.

\section{Tactile Pattern}

Tactile patterns can be made to evoke different sensations. In the current study, we define a tactile pattern as the combination of two components: its shape (i.e. form, path) and its sensation (i.e. feeling, perception).

Initial investigations on mid-air tactile patterns were concerned with how to produce a spatially distributed tactile 
sensation. For instance Wilson et al. [30] looked at the perception of mid-air tactile stimulus moving in a linear direction and across different distances. The importance of temporal parameters according to the desired travel distances was highlighted. The ability to produce multi-points to render 3D volumetric shapes was first leveraged and demonstrated in Long et al. [16]. Here, the desired shapes were sampled using several tactile points and modulating the amplitude of each point. Using a similar approach Korres et al. [15] investigate the effect of varying rendering parameters for circular tactile patterns. Therefore, most previous works concerned with tactile patterns mainly focused on the stimulation duration and the stimulus onset asynchrony. Moreover, most previous research has been using AM techniques since LM and STM are very recent findings. Nonetheless Frier et al. [5] show a strong effect of pattern draw frequency on the pattern perceived strength, with STM, and therefore recommend to optimise the pattern draw frequency according to pattern size.

An interesting property of mid-air tactile patterns is the ability to completely change the users perception of the haptic feedback by simply changing the modulation parameters. For instance Obrist et al. [19] conveyed different qualitative perception such as pulsing, prickly or dispersed, solely by changing AM stimulus modulation frequency. Monnai et al. [18] varied the whole modulation signal according to the application scenario, to provide use case specific tactile effect. In general, these tactile perceptions can either be discriminative and convey information such as the texture of a pattern [4], or be affective and convey emotion [20]. It is worth mentioning that to the best of our knowledge, none of those studies use modulation frequency lower than $16 \mathrm{~Hz}$.

The challenge of choosing the rendering parameters for a given tactile pattern is therefore to achieve this differences in both discriminative and affective perceptions. The combined requirements however can make this process complex and thus confusing. For application level HCI research such as for media studies [1] or art studies [2], it is easier if mid-air tactile patterns were implemented from a template library or available in the API implementation.

\section{SAMPLING STRATEGY}

With STM, a tactile pattern is produced using a single midair tactile point. Therefore, producing the pattern shape as well as its associated sensation requires rapid and repeated updates of the point properties. In this part, we discuss the update rate requirements and its relation to what we call the pattern sampling rate. Then, we present the current sampling strategy applied in the literature. Finally, we evaluate the current strategies in an effort to find a new, more optimal sampling strategy.

\section{Pattern Sampling Rate}

We saw in the previous section that a tactile pattern was composed of a shape and a sensation. However, tracing a pattern shape requires sampling the shape into a set of intermediate discrete positions, referred to as sample points (see Figure 1.a). When rendering the pattern shape, the tactile point position will thus be updated successively to each of those sample points position. The number of sample points, referred to as sampling rate, can vary. However, the greater the sampling rate, the smoother the pattern representation. For instance a circle traced with 20 points will appear smoother than a circle traced with 10 points (see Figure 1-b\&c).

Adjusting the tactile sensation is equivalent to tuning its modulation frequency [19]. The modulation frequency being the rate at which a specific skin patch is repeatedly stimulated, in the case of STM the modulation frequency is similar to the rate at which the pattern is drawn, referred to as draw frequency Indeed, drawing a circle at $5 \mathrm{~Hz}$, means tracing out the circle path 5 times per second and therefore stimulated each position along the circle 5 times per second. Therefore, adjusting the tactile sensation is equivalent to tuning its draw frequency.

Furthermore, if the circle sampling rate is of 10 points per circle, it means that the point will move through each 10 positions 5 times a second. In other words, the point position will be updated 50 times per second $(5 \mathrm{~Hz} \times 10$ points). Thus, a mid-air tactile display, updating a given pattern, needs an update rate equivalent to the product of the pattern sampling rate times the pattern modulation frequency (see equation 1 ).

$$
\text { update rate }=\text { sampling rate } \times \text { draw frequency }
$$

We would like to highlight that the update rate thus defined is not necessarily the maximum update rate that a mid-air tactile display can achieve. Therefore, for update rates lower than that achievable by the hardware, it is possible to increase either the pattern sampling rate or draw frequency without decreasing the other. However, at update rates close to hardware maximum capabilities, a trade-off between sampling rate and draw frequency is required.

\section{Current Sampling Strategies}

We define as sampling strategy the tuning of pattern sampling rates according to specific criteria. We found that all previous work use the same strategy that is to determine the pattern sampling rate as the maximum update rate the hardware can achieve, divided by the draw frequency. For instance, Kappus and Long [13] are producing a circle at $200 \mathrm{~Hz}$ using the full $20 \mathrm{kHz}$ update rate achievable by the hardware used in their experiments. We believe the reason is that the researchers assume that the greater the update rate the better. This assumption seems pertinent as it is a strategy already being used for different modalities. For instance, in 
vision and audio a higher sampling rate will reduce artefacts such as motion blur and flickering, for vision, and aliasing, for audio. We referred to this strategy as the high sampling rate strategy.

\section{Pros and Cons for High Sampling Rate Strategy}

A high sampling rate strategy can support high draw frequencies without distorting the tactile pattern shape. For example, a simple pattern shape such as a $150 \mathrm{~mm}$ circumference circle can be rendered at $500 \mathrm{~Hz}$ (the upper limit of vibrotactile frequency relevant to touch) using 40 sample points spaced out every $3.75 \mathrm{~mm}$. The spacing between two consecutive points being less than the tactile point radius ( $\approx 4.3 \mathrm{~mm}$ ), one would expect that the pattern will still be perceived as smooth and continuous.

However, the problem is that a high sampling rate strategy results in shorter stimulation durations. Indeed, increasing the pattern resolution decreases the relative stimulation duration of each point. For example, a device running at a update rate of $20 \mathrm{kHz}$, this means that the stimulation duration of one sample is $50 \mu \mathrm{s}$. This duration is 2 to 3 order of magnitude lower than the perceptible sense of touch temporal resolution found by Loomis [17] that was estimated to be between $2 \mathrm{~ms}$ and $40 \mathrm{~ms}$. Moreover, this draw frequency is 2 orders of magnitude higher than the vibrotactile frequency range relevant to our mechanoreceptors. It is therefore unlikely that our skin can perceived such rapid tactile stimuli.

Another challenge of using a higher sampling rate strategy is the possible inconsistency in sampling rate between tactile patterns being traced with different draw frequency.

The discussion thus far has therefore revealed that pattern variability demands customised pattern sampling rates, otherwise inherent variations will manifest themselves in an uncontrolled and misunderstood tactile perception. This paper will address the above mentioned challenges and propose mitigation strategies.

\section{USER STUDY 1}

There are various modulation methods and sampling strategies that can produce a mid-air tactile pattern using focused ultrasound. These methods and strategies predominantly depend on the available hardware being used. There has not been however any discussion on how sampling strategy affects the overall pattern perception. This section describes how we undertook to investigate the relation between sampling strategy and pattern perception. In particular, we focus on the pattern perceived strength relative to the pattern sampling rate.

\section{Method}

Our hypothesis was that the pattern sampling rate will have an effect on the perceived strength. To test this, we run a

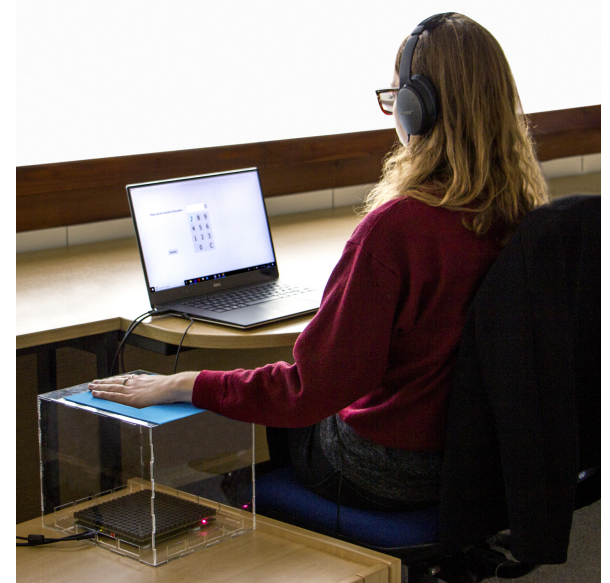

Figure 3: The set-up for the user studies. Participants were perceiving the mid-air tactile pattern on their left palm while rating each pattern on a designated laptop.

magnitude estimation task [11]. In this task, participants had to estimate the perceived strength for patterns rendered with different sampling rates.

In total 26 participants took part in the user study ( $6 \mathrm{fe}-$ males, average age \pm SD: $29.3 \pm 5.2$ ). Participants were sitting comfortably on an office chair, which they were free to adjust to their liking. On the left of the participant, there was an acrylic box, roughly at their hip level. The box was $200 \mathrm{~mm}$ high and a mid-air tactile display UHEV1 from Ultrahaptics Ltd. was lying at the bottom of the box. An aperture was cut on the top box, so participant can rest their left hand over it while experiencing the different mid-air tactile patterns. Before starting the study, an initial focal point was presented to the user hand, so they can align their palm with the array output. To avoid participants responses to be biased by surrounding noises, participants were wearing noise cancelling headphones which were playing pink noise. On the desk, in front of the participants, a laptop was running the experimental protocol. Participants could read instructions from the laptop screen and input their strength estimates via a computer mouse. Figure 3 shows the overall set-up.

To test our hypothesis we used a set of patterns with various sampling rates. To avoid shape related effects, all of these were variations on a circular pattern. All patterns were a $150 \mathrm{~mm}$ circumference circle (i.e. $\approx 24 \mathrm{~mm}$ centimetre radius), as it covers most of the palm of the participant (human palm width mostly varies between $75 \mathrm{~mm}$ and $95 \mathrm{~mm}$ [14]). Circles have also a clear periodic property and its intermediately positioned points can be easily made equally spaced, all of those limiting possible artefacts due to shape geometry. In this study, we also wanted to test whether the sampling rate of the pattern will affect the sensations of different patterns equally. Therefore we picked 6 different draw frequencies for 

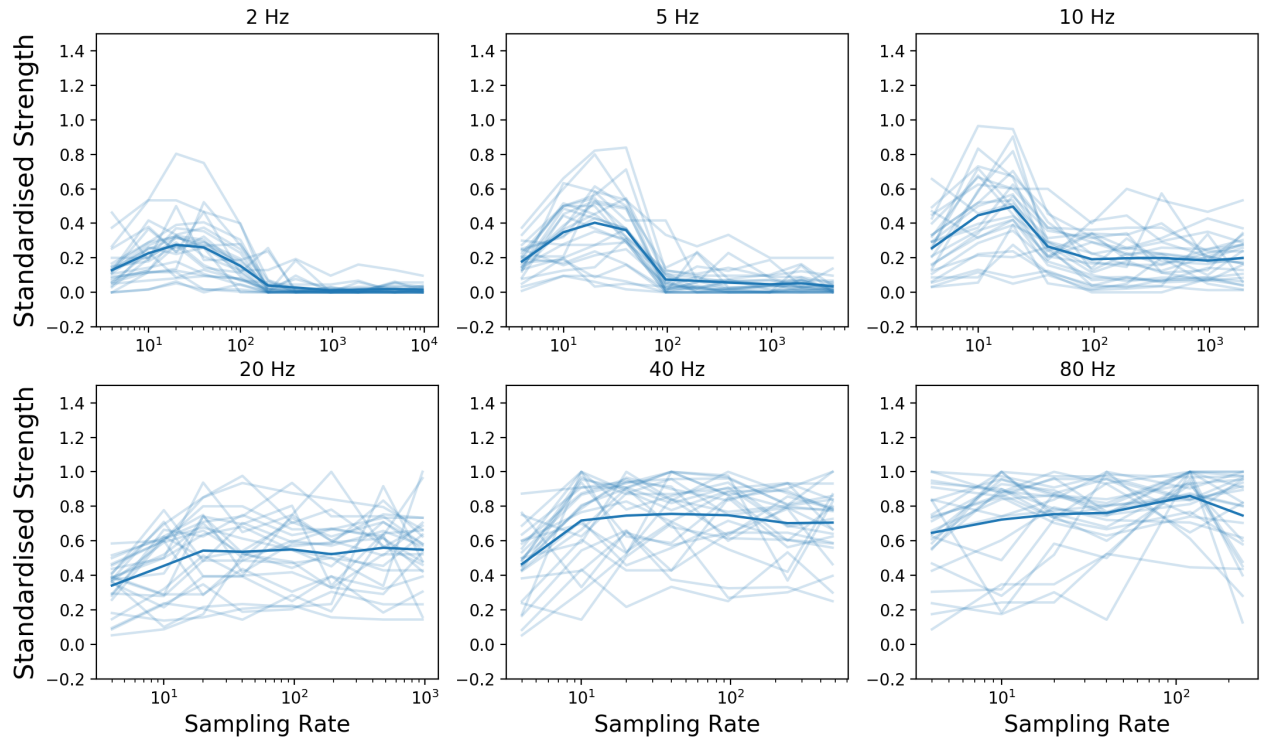

Figure 4: The standardise perceived strength as a function of the number of sampling points, for a $150 \mathrm{~mm}$ circumference circle rendered at different frequencies. Light and bold curves represent participants responses and responses average, respectively.

the presentation of the pattern, as to cover different octaves and the sensitivity ranges of different mechanoreceptors [10]. An illustration of such circular pattern is depicted in Figure 1-a, while Figure 1-b\&c show how the sampling rate affect the pattern spatiotemporal properties. The range of possible samples rates varied with the draw frequency. Due to this, we picked a total of 6 to 11 pattern sampling rates, depending on the draw frequency, which accounted for a total of 51 distinct patterns. Each pattern was repeated 3 times, making for a total of 153 stimuli in the study.

Each mid-air tactile pattern was presented to the participants left palm for 3 seconds. At the end of the stimulus, a numeric pad was displayed on the screen as well as an instruction inviting participants to enter their perceived strength estimates. Prior to the user study, participants were instructed to estimate the pattern perceived strength using their own scale. Additionally, participant were asked to rate the perceived strength from 0 as the minimum (i.e. did not feel the pattern), to infinite, using whole numbers (i.e. no decimal) and to be as consistent as possible in their estimation throughout the study. Finally, participants were reminded to focus only on the pattern perceived strength and to omit any other qualitative evaluation from their rating (e.g., smoothness or simultaneousness). After participants validated their response, the next pattern was presented after a two seconds break until participants rated all stimuli. The patterns order were presented in a randomised order. The whole study lasted about 20 minutes.
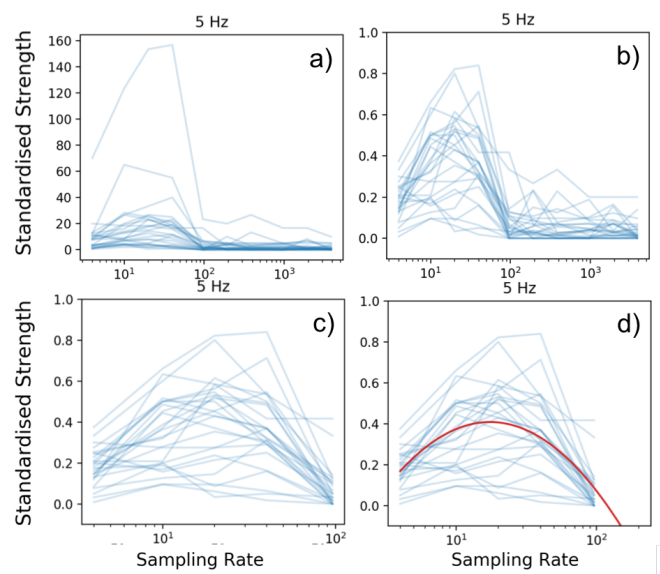

Figure 5: Data post-processing steps. (a) Raw data, (b) Standardised data, (c) Significant data, and (d) Fitted model.

\begin{tabular}{lrrr} 
& \multicolumn{3}{c}{ Quadratic Mixed Model } \\
\cline { 2 - 4 } Rate & $R^{2}$ & N. opt. & N. lim. \\
\hline $2 \mathrm{~Hz}$ & 0.68 & 22.4 & 236.6 \\
$5 \mathrm{~Hz}$ & 0.72 & 17.5 & 119.2 \\
$10 \mathrm{~Hz}$ & 0.62 & 15.8 & 149.4
\end{tabular}

Table 1: Quadratic mixed model results for frequency $2 \mathrm{~Hz}$, $5 \mathrm{~Hz}$ and $10 \mathrm{~Hz}$. Results include $R^{2}$, optimal sampling rate and sampling rate limit. 


\section{Results}

As participants were using different scale to estimate the pattern perceived strength, we first standardised the participants responses. That is, we divided each participant estimate by their highest response $[11,26]$. As we were interested in studying each pattern sensation separately, we further separated the data into 6 subsets, one for each pattern draw frequency. Post-processed participants perceived strength estimates are shown in Figure 4 as a function of pattern sampling rate. We invite the reader to note that the $x$-axis of the figure is logarithmically scaled as the pattern sampling rates spread across 4 orders of magnitude.

Each data subset was found to be unlikely to follow a normal distribution (Shapiro-Wilk, $p<.05$ ). Therefore we run a Friedman test on each data subset to test whether the perceived strength ratings were significantly different across sampling rate values. The Friedman test indicated significant differences between sampling rates groups for each draw frequency: $2 \mathrm{~Hz}\left(\chi^{2}(10)=199.1, p<.001\right), 5 \mathrm{~Hz}\left(\chi^{2}(9)=\right.$ $179.4, p<.001), 10 \mathrm{~Hz}\left(\chi^{2}(8)=109.9, p<.001\right), 20 \mathrm{~Hz}\left(\chi^{2}(7)=\right.$ $43.4, p<.001), 40 \mathrm{~Hz}\left(\chi^{2}(6)=45.6, p<.001\right)$ and $80 \mathrm{~Hz}\left(\chi^{2}(5)=\right.$ $15.26, p=.009)$.

To further determine whether the differences were significant across the whole range of sampling patterns, we run a pairwise Wilcoxon signed-rank test, with Bonferroni correction to avoid type 1 error. For draw frequencies $20 \mathrm{~Hz}$, $40 \mathrm{~Hz}$ and $80 \mathrm{~Hz}$, the Wilcoxon test indicated significant differences only between 1 or 2 pairs of sampling rates. We therefore discarded those draw frequencies for the end of the data analysis. However, for draw frequencies of $2 \mathrm{~Hz}, 5 \mathrm{~Hz}$ and $10 \mathrm{~Hz}$, the Wilcoxon test indicated significant differences for all sampling rate pairs, as long as the sampling rate was lower than 200, 96 and 48 points, respectively.

The fact that the upper sampling rate interval leads to no significant differences, suggest that no specific behaviour could be extracted from that part of the data. Furthermore, the fact the corresponding perceived strength plateau around 0 , suggest that the participants did not perceived those patterns. Those two points, motivated us to discard the data for the next step of the analysis and focus on the lower sampling rate interval.

On the remaining data, which correspond to the left part of the curve on Figure 4, the reader can see that, the pattern perceived strength seems to follow a quadratic behaviour. This apparent quadratic behaviour motivated us to use a quadratic linear model, to fit our data. The model we used for regression can be seen in equation 2

$$
\text { strength }=a \log _{10}^{2}(\text { sampling })+b \log _{10}(\text { sampling })+c
$$

We remind the reader, that the model uses logarithmic values as the plots on Figure 4, where the quadratic behaviour can be observed, are using logarithmic x-axes. The model gave $R^{2}$

\begin{tabular}{llccr} 
& & \multicolumn{3}{c}{ Quadratic Mixed Model } \\
\cline { 3 - 5 } Rate & Circum. & $R^{2}$ & N. opt. & N. lim. \\
\hline $2 \mathrm{~Hz}$ & $0.10 \mathrm{~m}$ & 0.69 & 18.72 & 240.55 \\
$2 \mathrm{~Hz}$ & $0.15 \mathrm{~m}$ & 0.60 & 20.95 & 241.76 \\
$2 \mathrm{~Hz}$ & $0.20 \mathrm{~m}$ & 0.61 & 27.95 & 260.29 \\
$10 \mathrm{~Hz}$ & $0.10 \mathrm{~m}$ & 0.67 & 10.77 & 92.09 \\
$10 \mathrm{~Hz}$ & $0.15 \mathrm{~m}$ & 0.60 & 15.01 & 90.54 \\
$10 \mathrm{~Hz}$ & $0.20 \mathrm{~m}$ & 0.60 & 20.30 & 84.52
\end{tabular}

Table 2: Quadratic mixed model results for frequency $2 \mathrm{~Hz}$ and $10 \mathrm{~Hz}$ across the different pattern sizes. Results include $R^{2}$, optimal sampling rate and sampling rate limit

values of $0.68,0.72$ and 0.63 for the pattern draw frequency $2 \mathrm{~Hz}, 5 \mathrm{~Hz}$ and $10 \mathrm{~Hz}$ respectively.

An $R^{2}$ value greater than 0.6 is considered high. We can therefore conclude that the quadratic model is a good fit to model our data. Hence we used the coefficient from the model to estimate the pattern sampling rate that was giving the highest perceived strength. We found that the optimal pattern sampling rate was $22.4,17.5$ and 15.8 points for draw frequency $2 \mathrm{~Hz}, 5 \mathrm{~Hz}$ and $10 \mathrm{~Hz}$, respectively. Finally we estimated the sampling rate threshold that was leading to the pattern to be perceived or not. We found a threshold of 236.6, 119.2 and 149.4 points for pattern draw frequency $2 \mathrm{~Hz}, 5 \mathrm{~Hz}$ and $10 \mathrm{~Hz}$, respectively. The post-processing step can be visualised in Figure 5 and the results of the data fitting are summarized in Table 1.

\section{USER STUDY 2}

In the first user study, we were able to determine a relation between pattern perceived strength and sampling rate. However this relation parameters are varying with the pattern draw frequency. In this second user study, we aim to determine whether these relation parameters vary as well when the pattern size changes.

\section{Method}

We use the same protocol and set-up as in the first study. The new stimuli set was composed of 2 draw frequencies. We chose $2 \mathrm{~Hz}$ and $10 \mathrm{~Hz}$, as they are the two boundary frequencies for which the effect of sampling strategy was observed in the first user study. There were 11 and 9 pattern sampling rates for the two draw frequencies, $2 \mathrm{~Hz}$ and $10 \mathrm{~Hz}$, respectively. We used 3 different pattern sizes, which were 100,150 and $200 \mathrm{~mm}$ circumference. There was a total of 60 distinct patterns. Each pattern was repeated 3 times, making a total of 180 stimuli. The user study lasted about 25 minutes. In total 26 participants (4 females, average age \pm SD: $30 \pm 5.9$ ) take part in the study. 

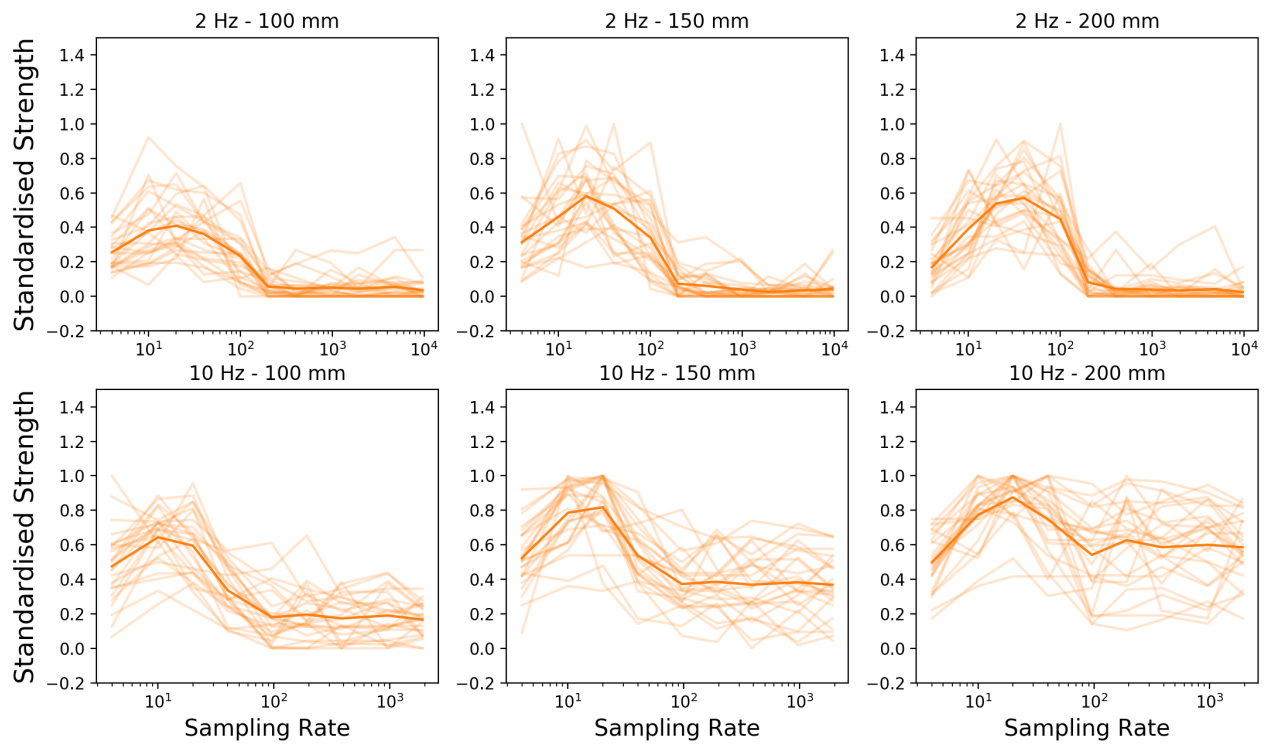

Figure 6: The standardised perceived strength as a function of the number of sampling points, for different frequencies and circle circumferences. Light curves represent participant responses and bold curves represent responses average.

\section{Results}

The data collected were standardised as in the first user study. We also separated the standardised responses into 6 subsets according to pattern draw frequency and pattern size. Figure 6 shows the resulting rating after standardisation for each data subset.

Each data subset was likely not normally distributed (ShapiroWilk, $p<.05)$.

Therefore, we run a Friedman test on each data set to test if the perceived strength rating were significantly different across the corresponding number of sampling rate. For patterns at $2 \mathrm{~Hz}$, Friedman test indicates significant differences as $\chi^{2}(10)=200.5, p<.001, \chi^{2}(10)=212.3, p<.001$ and $\chi^{2}(10)=208.9, p<.001$, for circumferences $100 \mathrm{~mm}$, $150 \mathrm{~mm}$ and $200 \mathrm{~mm}$, respectively. For pattern at $10 \mathrm{~Hz}$, Friedman test indicated significant differences as $\chi^{2}(8)=138.1, p<$ $.001, \chi^{2}(8)=111.0, p<.001$ and $\chi^{2}(10)=84.0, p<.001$, for circumferences $100 \mathrm{~mm}, 150 \mathrm{~mm}$ and $200 \mathrm{~mm}$, respectively. To further determine whether the differences were significant across the whole range of sampling pattern, we run a pairwise Wilcoxon signed-rank test, with Bonferroni correction to avoid type 1 error, on each data subset, and thus determine which pair of pattern sampling rate were significantly different. As in the first study, we found that the pairs of sampling rates were significantly different only for sampling rate below 200 and 96 points, for modulation $2 \mathrm{~Hz}$ and $10 \mathrm{~Hz}$, respectively. Hence, as for the same motivations as the first user study, we discarded the non-significant part of the data and run a quadratic mixed model on the significant part of the data. The model indicate $R^{2}$ values of $0.70,0.60$ and 0.61 for the pattern draw frequency $2 \mathrm{~Hz}$ and circumference $100 \mathrm{~mm}, 150 \mathrm{~mm}$ and $200 \mathrm{~mm}$, respectively. For pattern draw frequency of $10 \mathrm{~Hz}$ the model gave $R^{2}$ of $0.67,0.60$ and 0.60 for the patterns with circumference The model indicates $R^{2}$ values of $0.70,0.60$ and 0.61 for the pattern draw frequency $2 \mathrm{~Hz}$ and circumference $100 \mathrm{~mm}, 150 \mathrm{~mm}$ and $200 \mathrm{~mm}$, respectively.

An $R^{2}$ value greater than 0.6 is considered high. We can therefore conclude that the quadratic model is a good fit to model our data. Then we use the coefficients from the model to estimate the pattern sampling rate that was giving the highest perceived strength. We found that for $2 \mathrm{~Hz}$ draw frequency, the optimal sampling rate was 18.72, 20.95 and 27.95 points for circumference $100 \mathrm{~mm}, 150 \mathrm{~mm}$ and $200 \mathrm{~mm}$ respectively. We also found a sampling rate threshold of $240.55,241.76$ and 260.29 points for circumference 100, 150 and $200 \mathrm{~mm}$ respectively. For a draw frequency of $10 \mathrm{~Hz}$, we found an optimal sampling rate of 10.77, 15.01 and 20.30 points for circumference $100 \mathrm{~mm}, 150 \mathrm{~mm}$ and $200 \mathrm{~mm}$ respectively. As for $2 \mathrm{~Hz}$ modulation, the perceived strength of pattern at $10 \mathrm{~Hz}$ plateau when the sampling rate is greater than a given number. Using the model parameters and the plateau values, we found that the sampling rate threshold was $92.09,90.54$ and 84.52 for circumference $100 \mathrm{~mm}, 150 \mathrm{~mm}$ 
and $200 \mathrm{~mm}$ respectively. The results of the data fitting are summarized in Table 2.

\section{DISCUSSION}

In the current paper, we investigated a sampling strategy that maximised the pattern perceived strength. Using circular patterns rendered with different amounts of sampling points, we established a relationship between pattern sampling rate and pattern perceived strength. After discussing the user studies results, we will try to explain those same results using the psychophysical literature on the perception of touch. Finally, we will cover the implication of our work for tactile feedback designers.

\section{User studies Results}

In the two user studies, we demonstrated that pattern sampling rate has an effect on pattern perceived strength. However, significant effect was limited to patterns with draw frequencies ranging from $2 \mathrm{~Hz}$ to $10 \mathrm{~Hz}$. Although variability can be observed in user results magnitude, which could be accounted for user's subjective judgement, the overall trends are common across participants and can be modelled. Using a regression model, we successfully fitted the pattern perceived strength to a quadratic function of the logarithm of the sampling rate (see equation 2 ).

From these regression functions, we identified an optimal sampling rate for patterns rendered at $10 \mathrm{~Hz}$, of $10.77,15.01$ and 20.30 points for circumferences $100 \mathrm{~mm}, 150 \mathrm{~mm}$ and $200 \mathrm{~mm}$ respectively. By taking the ration of the pattern circumference over the optimal sampling rates, we obtained an optimal distance between sample points of $9.7 \mathrm{~mm} \pm 0.3$ The low variation between optimal distances between sample points, designate this distance as an invariant for maximising pattern perceived strength across pattern sizes. We found similar results with draw frequency of $2 \mathrm{~Hz}$, for which the optimal distance between samples points was in average equal to $6.5 \mathrm{~mm} \pm 0.8$. However, the optimal distances obtained are different across pattern draw frequency and despite our effort, we could not establish a clear relation between optimal distance and draw frequency.

Using the user study results, we also found that the perceived strength plateaus when the pattern sampling rate is greater than a given threshold. This threshold is in average $245 \pm 6.2$ points and $89 \pm 3.3$ points for patterns at $2 \mathrm{~Hz}$ and $10 \mathrm{~Hz}$, respectively. The low variation between threshold averages suggest the sampling rate threshold to be invariant across pattern sizes, although we could not establish the relation between threshold and pattern draw frequency.

Even though our study showed no effect of sampling rate on perceived strength for patterns at high frequency, we would like to point out that, when observed, the effect occurs only for sampling rate under 200 points. However, high frequency patterns can not currently be rendered with sampling rates up to 200 points. For instance, the mid-air tactile display we used could render a pattern at $80 \mathrm{~Hz}$ with only 24 points at most. It is likely that technology will improves and allow to render high frequency patterns with sampling rate of 200 points or more. Until then, we cannot completely ruled out the effect of sampling rate on perceived strength in the case of high frequency patterns.

Finally, on Figure 6, one may note that the maximum perceived strength vary with the pattern draw frequencies and sizes. However, this can be explained with Frier et al. [5] work, which claims that perceived strength varies with the tactile point speed (i.e. draw frequency times pattern circumference).

\section{Psychophysical Explanation}

In an attempt to further understand the results reported in this study, we discuss here some hypotheses related to the psychophysics of the sense of touch. Testing these hypotheses is beyond the present scope of this paper, yet we believe it could be informative towards the reader.

Firstly recall that, for AM, different modulation frequencies are perceived with different strength, even though the amplitude of the stimulation remains the same, $200 \mathrm{~Hz}$ being the frequency perceived the strongest [6].

However, STM stimulation can no longer be described as a sinusoid like for AM and LM, but more like a pulse train with alternation between intervals of stimulation and nonstimulation. Using a Fourier expansion, this pulse train can be decomposed as a sum of sinusoidal signals, thus unveiling the presence of harmonics that are higher in frequency, with an amplitude depending on the pulse width. Decreasing the sampling rate may inadvertently increase the harmonic's amplitude close to $200 \mathrm{~Hz}$, and thereby increase the associated perceived strength.

Another hypothesis is related to the skin viscoelastic properties. High sampling rate stimulation leads to stimulation durations being too short for the skin deformation to reach the required mechanoreceptors depth. At first, this hypothesis might seem unlikely since higher frequency patterns yield to tactile perception nonetheless. However by definition the rate at which the stimulation is repeated at a single location is much faster for high draw frequencies than for low draw frequencies. Therefore, it is plausible that at high frequencies the skin indentation builds up as the pattern is repeated over and over again whereas at low frequencies the elastic skin relaxes entirely between stimulation intervals.

Until now, mid-air haptics was relying on stimulating RA and PC mechanoreceptors that are sensitive to vibrations higher in frequency than the one involved in this study [10]. However, one could note that as the tactile points moves 
across the skin surface, different groups of SA1 mechanoreceptors might be stimulated. Indeed, SA1 mechanoreceptors are mostly sensitive to the stimulus onset and offset (i.e. transient stimulus). Therefore, as the mid-air stimulus moves from one position to another, the stimulus is offset at the old position and onset at the new position. However, when a sampling rate is too high the sample position difference is lower than SA1 receptive field [29], and do not lead to this transient behaviour and therefore to tactile perception.

Ultimately, using a mechanotransduction model as the one presented by Saal et al. [23], one could test some of these hypotheses. Although, such models only predict stimulus detection, but will not determine optimal stimulation.

\section{Haptic Implications}

Higher sampling rate does not always improve tactile perception and quite often, the old cliche is true: less is more. Such design insights can be hugely beneficial to haptic engineers, developers and designers. Using the general trend found in the user-study results, we've therefore proposed ways and relationships for such parameters and variations to be hidden behind easy-to-use software packages.

First, we encourage tactile feedback designers working with mid-air tactile display to decrease the sampling rate whenever rendering tactile pattern with low frequency. Decreasing the sampling rate for a sensation that initially cannot be perceived, might suddenly unlock the said sensation. For instance, circular patterns as studied here, could not be perceived below $20 \mathrm{~Hz}$ with a high sampling rate. However, when the sampling rate was lowered, the same circular pattern could be perceived as low as $2 \mathrm{~Hz}$. We would like to emphasise that since tactile perception of frequency follows a Weber-law, the range $2-10 \mathrm{~Hz}$ is half as wide as the range 10$200 \mathrm{~Hz}$. Hence increasing by $50 \%$ the range of discriminable frequency one could now apply to mid-air tactile patterns. We also would like to remind our readers, that in our study, we consider low frequency any frequency less than or equal to $10 \mathrm{~Hz}$. However our study focusing only on circular patterns, the $10 \mathrm{~Hz}$ frequency threshold might vary for other patterns, and hence ask the reader to interpret the values of this study carefully when applied to different shapes.

Then, we would like to invite feedback designers to adjust the sampling rate of a given mid-air tactile pattern, whenever it is possible, in order to maximise its perceived strength We also remind designers that this optimal sampling rate is proportional to the pattern size. Hence, when scaling a given pattern, the sampling rate should be scaled accordingly.

As no previous work exploring adjusting sampling strategy has been undertaken, we expect the possibility to render low frequency pattern to be unveiled for most designers working with mid-air tactile display. Moreover, low frequency patterns, operating at much lower speed than usual pattern rendered with STM, are now expected to be perceived as moving points rather than complete shapes. Moving points, providing richer information (such as start \& end locations, direction of motion and rate of movement, all of which are masked at higher speeds), are better recognised than multi-points pattern. This has already been demonstrated for contact devices by Ion et al. [8] who used unistroke patterns. We believe the distinction to hold between low and high frequency mid-air tactile patterns too. Hence, thanks to the sampling strategy we presented, a new horizon of possibilities has been made available to the designers. We encourage designers to experiment with and investigate those new possibilities.

Finally, we are conscious all the results presented here could be overwhelming for tactile feedback designers. However, the invariants identified in the second user study should ease the implementation of our results into design tools as hidden parameters. Thus improved design tools will allow current tactile feedback designers to stay oblivious to sampling strategies.

\section{CONCLUSION AND FUTURE WORKS}

Ultrasound phased arrays leverage high update rate to produce tactile patterns of different shapes and sensations. Yet, we show in the current study that in some cases sampling a pattern at high rate can be detrimental for pattern perception. Indeed, for low draw frequency patterns (from $2 \mathrm{~Hz}$ to $10 \mathrm{~Hz}$ ) the perceived strength of the pattern was significantly dependent on the sampling rate. However, our user studies identify estimated optimal sampling rate, as well as rules to adapt those optimum across different pattern size. We then discuss how these results could benefit mid-air tactile feedback designers. Especially, if those results could be integrated into a designer tool, in order to keep designers oblivious to the complex sampling strategies.

In future works, we will continue our investigation on the effect of sampling rate on pattern perception. Particularly, we will focus on expressing the optimum sampling rate as a function of pattern draw frequency and pattern shape. The potential outcomes of these future works will serve to expand the current guidelines for tactile pattern designers. Finally, further future works will consider applying the current guidelines to produce more complex pattern as well as integrating those new patterns into a variety of application scenarios for mid-air interactions.

\section{ACKNOWLEDGMENTS}

This project has received funding from Ultrahaptics and the European Union's Horizon 2020 research and innovation programmes under the FET-Open grant schemes (Levitate-No 737087 and H-Reality-No 801378) and the European Research Council (ERC) under grant No 638605. 


\section{REFERENCES}

[1] Damien Ablart, Carlos Velasco, and Marianna Obrist. 2017. Integrating mid-air haptics into movie experiences. TVX 2017 - Proceedings of the 2017 ACM International Conference on Interactive Experiences for TV and Online Video (2017). https://doi.org/10.1145/3077548.3077551

[2] Maryam Azh, Shengdong Zhao, and Sriram Subramanian. 2016. Investigating Expressive Tactile Interaction Design in Artistic Graphical Representations. ACM Transactions on Computer-Human Interaction 23, 5 (2016), 1-47. https://doi.org/10.1145/2957756

[3] Tom Carter, Sue Ann Seah, Benjamin Long, Bruce Drinkwater, and Sriram Subramanian. 2013. UltraHaptics : Multi-Point Mid-Air Haptic Feedback for Touch Surfaces. (2013).

[4] Euan Freeman, Ross Anderson, Julie Williamson, Graham Wilson, and Stephen Brewster. 2017. Textured Surfaces for Ultrasound Haptic Displays. Proceedings of the 19th ACM International Conference on Multimodal Interaction - ICMI '17 Demos (2017), 7-8. https://doi.org/ 10.1145/3136755.3143020

[5] William Frier, Damien Ablart, Jamie Chilles, Benjamin Long, Marcello Giordano, Marianna Obrist, and Sriram Subramanian. 2018. Using Spatiotemporal Modulation to Draw Tactile Patterns in Mid-Air. 270281. https://doi.org/10.1007/978-3-319-93445-7\{_\}24

[6] G. a. Gescheider, S. J. Bolanowski, and R. T. Verrillo. 2004. Some characteristics of tactile channels. Behavioural Brain Research 148, 1-2 (2004), 35-40. https://doi.org/10.1016/S0166-4328(03)00177-3

[7] Takayuki Hoshi, Masafumi Takahashi, Takayuki Iwamoto, and Hiroyuki Shinoda. 2010. Noncontact tactile display based on radiation pressure of airborne ultrasound. IEEE Transactions on Haptics 3, 3 (2010), 155-165. https://doi.org/10.1109/TOH.2010.4

[8] Alexandra Ion, Edward Jay Wang, and Patrick Baudisch. 2015. Skin Drag Displays. In Proceedings of the 33rd Annual ACM Conference on Human Factors in Computing Systems - CHI '15. ACM Press, New York, New York, USA, 2501-2504. https://doi.org/10.1145/2702123.2702459

[9] Mitsuru Ito, Daisuke Wakuda, Seki Inoue, Yasutoshi Makino, and Hiroyuki Shinoda. 2016. High Spatial Resolution Midair Tactile Display Using $70 \mathrm{kHz}$ Ultrasound. 57-67. https://doi.org/10.1007/ 978-3-319-42321-0\{_\}6

[10] Roland S. Johansson and J. Randall Flanagan. 2009. Coding and use of tactile signals from the fingertips in object manipulation tasks. Nature reviews. Neuroscience 10, 5 (2009), 345-359. https://doi.org/10.1038/ nrn2621

[11] Lynette A Jones, Senior Member, Hong Z Tan, and Senior Member. 2013. Application of Psychophysical Techniques to Haptic Research. 6, 3 (2013), 268-284.

[12] Jae-Hoon Jun, Jong-Rak Park, Sung-Phil Kim, Young Min Bae, JangYeon Park, Hyung-Sik Kim, Seungmoon Choi, Sung Jun Jung, Seung Hwa Park, Dong-Il Yeom, Gu-In Jung, Ji-Sun Kim, and Soon-Cheol Chung. 2015. Laser-induced thermoelastic effects can evoke tactile sensations. Scientific Reports 5 (2015), 11016. https://doi.org/10.1038/ srep11016

[13] Brian Kappus and Benjamin Long. [n. d.]. SPATIOTEMPORAL MODULATION FOR MID-AIR HAPTIC FEED- BACK FROM AN ULTRASONIC PHASED ARRAY. 1 ([n. d.]), 1-6.

[14] Sashidharan Komandur, Peter W. Johnson, Richard L. Storch, and Michael G. Yost. 2009. Relation between index finger width and hand width anthropometric measures. Proceedings of the 31st Annual International Conference of the IEEE Engineering in Medicine and Biology Society: Engineering the Future of Biomedicine, EMBC 2009 (2009), 823826. https://doi.org/10.1109/IEMBS.2009.5333195

[15] Georgios Korres, Tamas Aujeszky, and Mohamad Eid. 2017. Characterizing tactile rendering parameters for ultrasound based stimulation. In 2017 IEEE World Haptics Conference (WHC). IEEE, 293-298.
https://doi.org/10.1109/WHC.2017.7989917

[16] Benjamin Long, Sue Ann Seah, Tom Carter, and Sriram Subramanian. 2014. Rendering volumetric haptic shapes in mid-air using ultrasound. ACM Transactions on Graphics 33, 6 (2014), 1-10. https://doi.org/10. $1145 / 2661229.2661257$

[17] J. M. Loomis. 1981. Tactile pattern perception. Perception 10, 1 (1981), 5-27. https://doi.org/10.1068/p100005

[18] Yasuaki Monnai, Keisuke Hasegawa, Masahiro Fujiwara, Kazuma Yoshino, Seki Inoue, and Hiroyuki Shinoda. 2014. HaptoMime : MidAir Haptic Interaction with a Floating Virtual Screen. Uist '14 (2014), 663-667. https://doi.org/10.1145/2642918.2647407

[19] Marianna Obrist, Sue Ann Seah, and Sriram Subramanian. 2013. Talking About Tactile Experiences. Proceedings of the SIGCHI Conference on Human Factors in Computing Systems (2013), 1659-1668. https://doi.org/10.1145/2470654.2466220

[20] Marianna Obrist, Sriram Subramanian, Elia Gatti, Benjamin Long, and Thomas Carter. 2015. Emotions Mediated Through Mid-Air Haptics. Proc. CHI'15 (2015), 2053-2062. https://doi.org/10.1145/2702123. 2702361

[21] Yoichi Ochiai, Kota Kumagai, Takayuki Hoshi, Satoshi Hasegawa, and Yoshio Hayasaki. 2016. Cross-Field Aerial Haptics : Rendering Haptic Feedback in Air with Light and Acoustic Fields. Chi '16 (2016), 32383247. https://doi.org/10.1145/2858036.2858489

[22] Yoichi Ochiai, Kota Kumagai, Takayuki Hoshi, Jun Rekimoto, Satoshi Hasegawa, and Yoshio Hayasaki. 2015. Fairy Lights in Femtoseconds: Aerial and Volumetric Graphics Rendered by Focused Femtosecond Laser Combined with Computational Holographic Fields. (2015). http: //arxiv.org/abs/1506.06668

[23] Hannes P. Saal, Benoit P. Delhaye, Brandon C. Rayhaun, and Sliman J. Bensmaia. 2017. Simulating tactile signals from the whole hand with millisecond precision. Proceedings of the National Academy of Sciences 114, 28 (2017). https://doi.org/10.1073/pnas.1704856114

[24] Rajinder Sodhi, I Poupyrev, M Glisson, and A Israr. 2013. AIREAL: interactive tactile experiences in free air. SIGGRAPH (2013). http: //dl.acm.org/citation.cfm?id=2462007

[25] Daniel Spelmezan. 2016. Sparkle : Towards Haptic Hover-Feedback with Electric Arcs. (2016), 55-57.

[26] Paul Strohmeier and Kasper Hornbæk. 2017. Generating Haptic Textures with a Vibrotactile Actuator. Proc. CHI '17 (2017). https: //doi.org/10.1145/3025453.3025812

[27] Ryoko Takahashi, Keisuke Hasegawa, and Hiroyuki Shinoda. 2018. Lateral Modulation of Midair Ultrasound Focus for Intensified Vibrotactile Stimuli. Lecture Notes in Computer Science (including subseries Lecture Notes in Artificial Intelligence and Lecture Notes in Bioinformatics) 10894 LNCS (2018), 276-288. https://doi.org/10.1007/978-3-319-93399-3\{_\}25

[28] Mohamed Yassine Tsalamlal, Paul Issartel, Nizar Ouarti, and Mehdi Ammi. 2014. HAIR: HAptic feedback with a mobile AIR jet. Proceedings - IEEE International Conference on Robotics and Automation (2014), 26992706. https://doi.org/10.1109/ICRA.2014.6907246

[29] A B Vallbo and Roland S. Johansson. 1984. Properties of cutaneous mechanoreceptors in the human hand related to touch sensation. $\mathrm{Hu}$ man neurobiology 3, 1 (1984), 3-14.

[30] Graham Wilson, Thomas Carter, Sriram Subramanian, and Stephen A. Brewster. 2014. Perception of ultrasonic haptic feedback on the hand. In Proceedings of the 32nd annual ACM conference on Human factors in computing systems - CHI '14. ACM Press, New York, New York, USA, 1133-1142. https://doi.org/10.1145/2556288.2557033 